\title{
Refractive index dispersion sensing using an array of photonic crystal resonant reflectors
}

Hermannsson, Pétur Gordon; Vannahme, Christoph; Smith, Cameron; Sørensen, Kristian Tølbøl; Kristensen, Anders

Published in:

Applied Physics Letters

Link to article, DOI:

$10.1063 / 1.4928548$

Publication date:

2015

Document Version

Publisher's PDF, also known as Version of record

Link back to DTU Orbit

Citation (APA):

Hermannsson, P. G., Vannahme, C., Smith, C., Sørensen, K. T., \& Kristensen, A. (2015). Refractive index dispersion sensing using an array of photonic crystal resonant reflectors. Applied Physics Letters, 107(6), [061101]. https://doi.org/10.1063/1.4928548

\section{General rights}

Copyright and moral rights for the publications made accessible in the public portal are retained by the authors and/or other copyright owners and it is a condition of accessing publications that users recognise and abide by the legal requirements associated with these rights.

- Users may download and print one copy of any publication from the public portal for the purpose of private study or research.

- You may not further distribute the material or use it for any profit-making activity or commercial gain

- You may freely distribute the URL identifying the publication in the public portal 


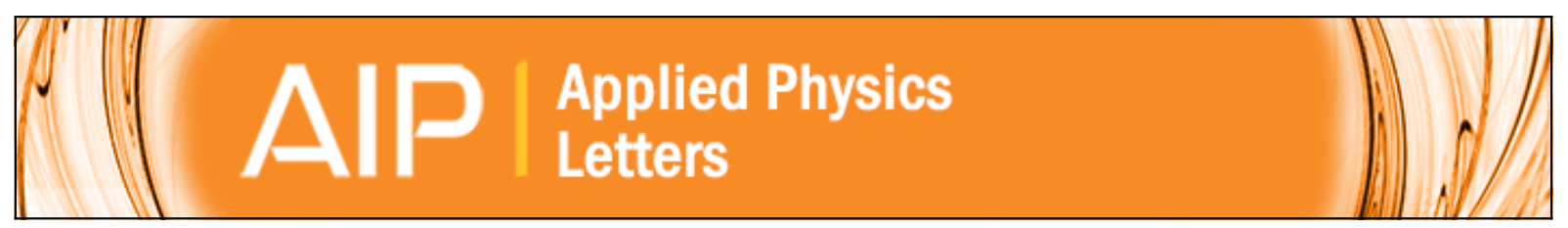

\section{Refractive index dispersion sensing using an array of photonic crystal resonant reflectors}

Pétur G. Hermannsson, Christoph Vannahme, Cameron L. C. Smith, Kristian T. Sørensen, and Anders

Kristensen

Citation: Applied Physics Letters 107, 061101 (2015); doi: 10.1063/1.4928548

View online: http://dx.doi.org/10.1063/1.4928548

View Table of Contents: http://scitation.aip.org/content/aip/journal/apl/107/6?ver=pdfcov

Published by the AIP Publishing

\section{Articles you may be interested in}

Photonic crystal slot nanobeam slow light waveguides for refractive index sensing

Appl. Phys. Lett. 97, 151105 (2010); 10.1063/1.3497296

Photonic crystal heteroslab-edge microcavity with high quality factor surface mode for index sensing

Appl. Phys. Lett. 94, 141110 (2009); 10.1063/1.3117225

Ultrastable in reflection photonic crystal fiber modal interferometer for accurate refractive index sensing Appl. Phys. Lett. 93, 191106 (2008); 10.1063/1.3025576

Silicon photonic crystal nanostructures for refractive index sensing

Appl. Phys. Lett. 93, 181103 (2008); 10.1063/1.3009203

Direct measurement of the group index of photonic crystal waveguides via Fourier transform spectral interferometry

Appl. Phys. Lett. 90, 261107 (2007); 10.1063/1.2752761

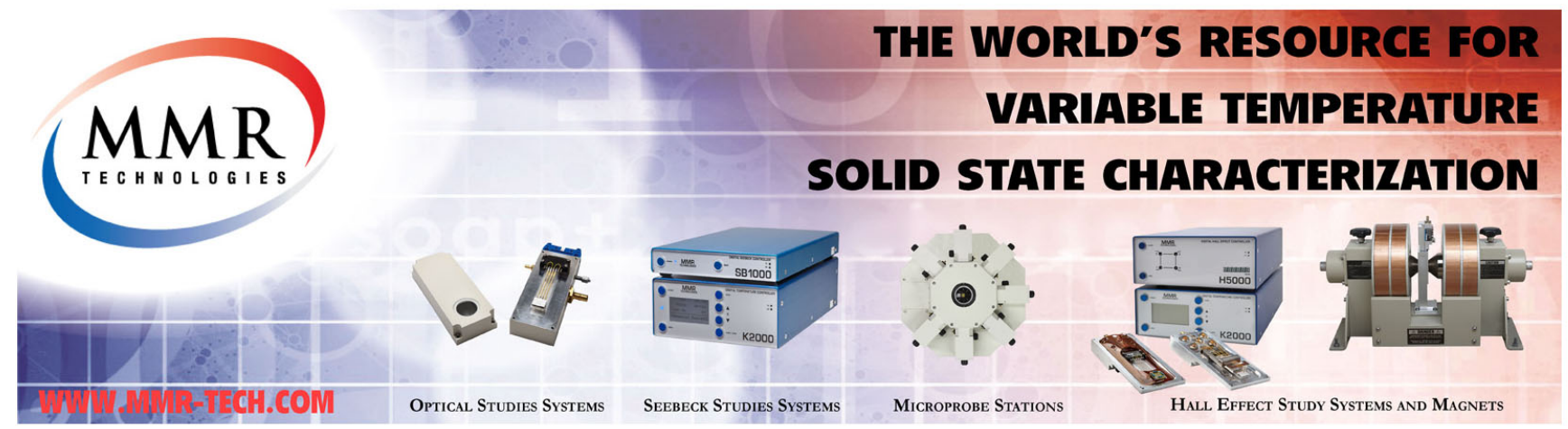




\title{
Refractive index dispersion sensing using an array of photonic crystal resonant reflectors
}

\author{
Pétur G. Hermannsson, Christoph Vannahme, Cameron L. C. Smith, Kristian T. Sørensen, \\ and Anders Kristensen ${ }^{\text {a) }}$ \\ Department of Micro- and Nanotechnology, Technical University of Denmark, Ørsteds Plads, Building 345E, \\ DK-2800 Kgs. Lyngby, Denmark
}

(Received 29 June 2015; accepted 2 August 2015; published online 12 August 2015)

\begin{abstract}
Refractive index sensing plays a key role in various environmental and biological sensing applications. Here, a method is presented for measuring the absolute refractive index dispersion of liquids using an array of photonic crystal resonant reflectors of varying periods. It is shown that by covering the array with a sample liquid and measuring the resonance wavelength associated with transverse electric polarized quasi guided modes as a function of period, the refractive index dispersion of the liquid can be accurately obtained using an analytical expression. This method is compact, can perform measurements at arbitrary number of wavelengths, and requires only a minute sample volume. The ability to sense a material's dispersion profile offers an added dimension of information that may be of benefit to optofluidic lab-on-a-chip applications. (C) 2015 AIP Publishing LLC. [http://dx.doi.org/10.1063/1.4928548]
\end{abstract}

In the preceding decades, various miniaturized optical refractive index (RI) sensors based on, e.g., surface plasmon resonance, ring resonators, optical fibers, and photonic crystals have been demonstrated, ${ }^{1}$ whose key advantage is the minimal amount of sample material required for analysis. In these sensors, a change in RI of the analyte material is registered via a change in the transduced output signal; however, the absolute value of the RI is typically not arrived at. Furthermore, the RI of the sample is only probed at a single wavelength, thereby neglecting the potentially valuable information involved in RI dispersion. In this paper, a method for measuring the absolute RI dispersion of liquids using an array of photonic crystal resonant reflectors (PCRRs) of varying periods is presented, which has traditionally required the use of the Newton-Fraunhofer method of minimum deviation. $^{2}$ The presented method is compact in nature, can perform measurements at an arbitrary number of wavelengths, requires only a minute sample volume, and is thus wellsuited for optofluidic lab-on-a-chip applications.

PCRRs, also known as guided-mode resonance filters or reflectors, ${ }^{3}$ and photonic crystal slabs, ${ }^{4}$ are optical structures capable of efficiently reflecting a given wavelength interval of out-of-plane incident light. These structures are composed of a periodically modulated high RI dielectric waveguide layer clad in media of lower refractive indices. When illuminated with broad-band light, guided resonances are excited within the waveguide layer, which are quasi-guided modes with finite lifetimes that couple to the far field. ${ }^{4,5}$ The modes are continually de-coupled out of the waveguide and interfere with both the transmitted and reflected parts of the incident light. At a certain resonance condition, destructive interference occurs between the de-coupled light and the transmitted light, leading to efficient resonant reflection, the linewidth of which is inversely proportional to the resonance lifetime. Depending on whether the incident light is polarized perpendicular or parallel to the

a) anders.kristensen@nanotech.dtu.dk. URL: www.nanotech.dtu.dk/ak grating direction, either transverse-electric (TE) or transversemagnetic (TM) quasi guided modes will be excited, respectively, and each mode will give rise to resonant reflection. ${ }^{5}$ Numerous applications of PCRRs have been suggested and demonstrated, ${ }^{6-9}$ as well as having made strong inroads into the fields of biological sensing ${ }^{10,11}$ and surface imaging. ${ }^{12,13}$

Fig. 1(a) shows a schematic illustration of a single PCRR used for the purpose of RI dispersion sensing. It is a

(a)

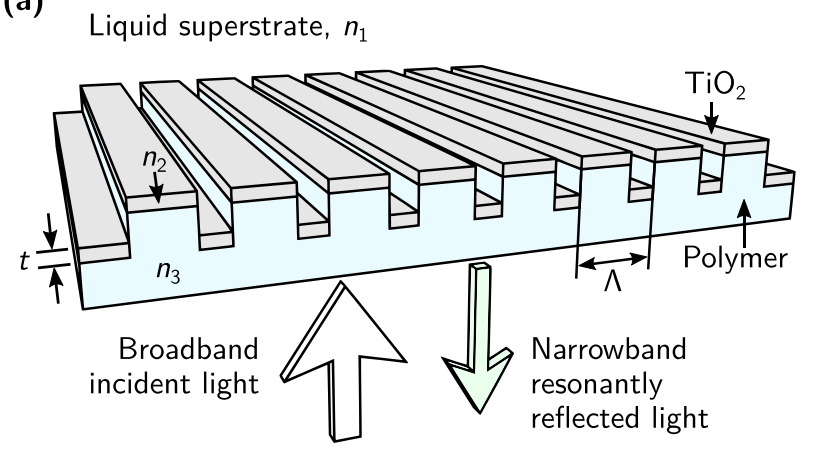

(b)

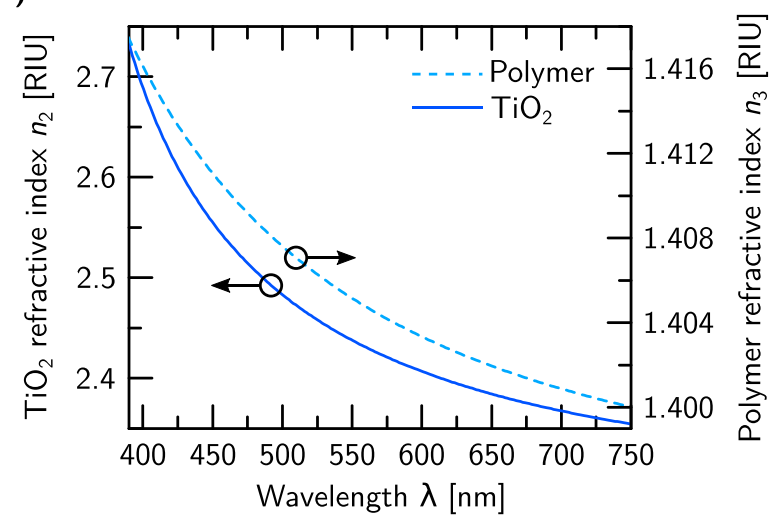

FIG. 1. (a) Schematic illustration of a photonic crystal resonant reflector and its operation. (b) The RI dispersion profiles of the two materials comprising the PCRRs investigated here. 
three layer dielectric structure composed of a periodically modulated high RI layer $\left(n_{2}\right)$, supported by a polymer substrate $\left(n_{3}\right)$, and covered with a liquid superstrate $\left(n_{1}\right)$, the RI dispersion of which is to be measured. The period of the modulation is $\Lambda$, and the thickness of the high RI layer is $t$.

It has been shown previously that the resonance wavelengths of resonant reflections exhibited by PCRRs can be accurately predicted by modeling the devices as a dielectric slab waveguides in which the propagation constant $\beta$ is determined by a phase-matching condition, ${ }^{14,15}$ which, for the case of normally incident light is given by

$$
\beta=\frac{2 \pi}{\Lambda},
$$

and is often referred to as the second-order Bragg condition. For the case of TE-polarized modes in a slab waveguide, the following mode condition must be fulfilled ${ }^{16}$

$$
\tan (h t)=\frac{p+q}{h-p q / h},
$$

where

$$
\begin{aligned}
& q=\left[\beta^{2}-n_{1}^{2} k_{0}^{2}\right]^{1 / 2}, \\
& h=\left[n_{2}^{2} k_{0}^{2}-\beta^{2}\right]^{1 / 2}, \\
& p=\left[\beta^{2}-n_{3}^{2} k_{0}^{2}\right]^{1 / 2},
\end{aligned}
$$

and by solving Eqs. (1) and (2) for $k_{0}$, the resonance wavelength $\lambda_{\mathrm{R}}$ can be obtained via $\lambda_{\mathrm{R}}=2 \pi / k_{0}$. Conversely, Eqs. (1)-(3) can be combined to yield the RI of the superstrate in terms of the resonance wavelength as follows:

$$
n_{1}\left(\lambda_{\mathrm{R}}\right)=\frac{1}{k_{0}} \sqrt{\frac{4 \pi^{2}}{\Lambda^{2}}-\left(\frac{h \tan (h t)-p}{1+p \tan (h t) / h}\right)^{2}},
$$

where $k_{0}=2 \pi / \lambda_{\mathrm{R}}$, and $h, p$ are functions of $\lambda_{\mathrm{R}}$. When a PCRR exhibits resonant reflection at a given wavelength $\lambda_{R}$, the light is effectively experiencing the refractive indices of the materials comprising the device and superstrate at that particular wavelength. Thus, if the refractive indices $n_{2,3}$ at this wavelength are known, as well as the structural parameters $\Lambda$ and $t$, the RI of the superstrate $n_{1}$ at $\lambda_{\mathrm{R}}$ can be calculated using Eq. (6). Utilizing the fact that $\lambda_{\mathrm{R}}$ is a monotonously increasing function of the grating period $\Lambda,{ }^{15}$ an array of PCRRs with varying grating periods $\Lambda$ can thus be established such that the resonances span the visible optical regime. By measuring the resonance wavelength exhibited by each PCRR and calculating the corresponding values of $n_{1}\left(\lambda_{\mathrm{R}}\right)$, the RI dispersion of the superstrate can hence be obtained.

For this purpose, an array of PCRRs with grating periods equispaced in the range $\Lambda=(260-470) \mathrm{nm}$ was fabricated using UV nanoreplication (see Ref. 15 for details). The PCRRs were composed of a low RI polymer substrate (Efiron PC-409, SSCP Co., Ltd) with a surface height modulation of $100 \mathrm{~nm}$ and a protrusion duty cycle of $75 \%$, covered with a $t \approx 28 \mathrm{~nm}$ thick layer of ion-beam deposited titanium dioxide. The RI dispersion profiles of the polymer and $\mathrm{TiO}_{2}$ were obtained by fitting variable angle spectroscopic ellipsometry data to a Cauchy and Tauc-Lorentz oscillator model,${ }^{17}$ respectively (Fig. 1(b)).

The wavelength spectra of the resonant reflections associated with TE-polarized quasi guided modes were measured for each of the PCRRs in the fabricated array for several superstrate liquids by illumination at normal incidence from the back-side, using the setup shown in Fig. 2(a). Here, light from a spectrally smooth and broadband light source (Energetiq EQ-99XFC) is fed to the setup via a multimode optical fiber and collimated with a fiber collimator. An image of the fiber tip is then focused and reflected into the image plane of a microscope objective by a lens and 50:50 beamsplitter, respectively. This causes the light to emerge collimated from the other side of the objective and illuminate the PCRR at normal incidence. The reflected light travels backwards through the objective and beamsplitter until it encounters a polarizing beamsplitter, where light associated with the TE-polarized modes is reflected to a spectrometer (PI Acton SP2750), and light associated with TM-polarized modes is transmitted. This allows for selective measurement of TE resonances and eliminates the TM resonances' contribution to the acquired signal noise. The spectrometer contains a diffraction grating with 1800 lines/mm and projects the input spectrum onto a CCD camera (PI Pixis 100) with a resolution of $12 \mathrm{pm} /$ pixel. The camera is further connected to

(a)

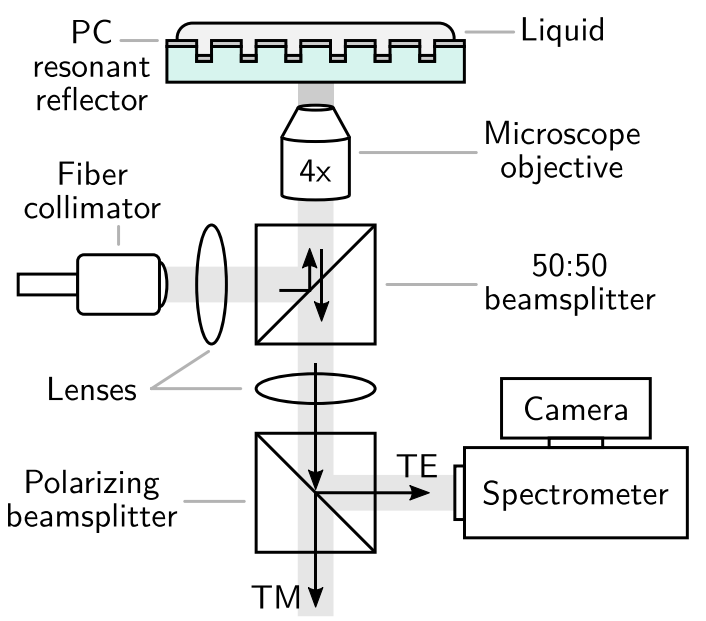

(b)

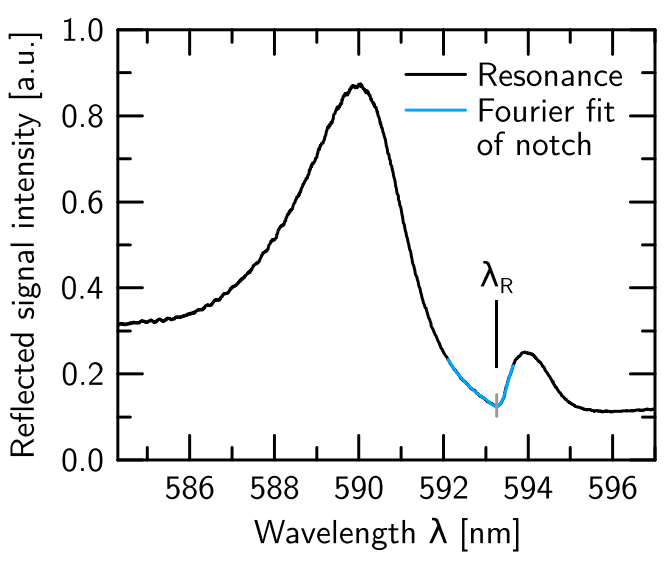

FIG. 2. (a) The experimental setup used for measuring the wavelength spectra of resonantly reflected light associated with TE-polarized quasi guided modes. (b) An example of such an acquired spectrum for the case of $\Lambda=386 \mathrm{~nm}$ and a glycerol liquid superstrate. 
a computer, which records the spectrum. The spectrometer was calibrated in advance with respect to a helium and neonargon calibration source. An example of an acquired resonance spectrum is shown in Fig. 2(b). The resonance spectra are characterized by a pair of intensity peaks separated by a dip, or notch. The peaks and notch correspond to the edges of two anticrossing photonic bands and the band gap between them, respectively. ${ }^{18}$ The wavelength minimum of the notch corresponds to the resonance wavelength $\lambda_{R}$ in the model. The notch is a consistent, narrow, and clear spectral feature, and is thus advantageous for measuring the resonance wavelength.

Reflected resonance spectra were acquired as a function of period $\Lambda$ for four different superstrate liquids: purified water, a RI matching oil (AAA 1.3850, Cargille Labs), glycerol (Sigma-Aldrich), and a microscope immersion oil (Type LDF, Cargille Labs). Upon spectral acquisition, the temperatures of the liquids as determined by a thermocouple were as follows: $21^{\circ} \mathrm{C}$ in the case of the two oils, $20^{\circ} \mathrm{C}$ in the case of water due to evaporative cooling, and $23.5^{\circ} \mathrm{C}$ in the case of glycerol due to optical heating. For each measurement, the notch was fitted with a four-term Fourier series (10 fitting parameters), from which the wavelength at the intensity minimum was obtained to yield the resonance wavelength $\lambda_{R}$, as illustrated in Fig. 2(b).

The purpose of the purified water was to serve as a reference liquid to which the PCRR array could be calibrated, and was selected for this function on account of its RI dispersion being accurately known across the entire visible wavelength regime at various temperatures. Using the experimentally obtained resonance wavelengths $\lambda_{R}$ as a function of period $\Lambda$ for the case of purified water, the RI values as yielded by Eq. (6) were fitted via the thickness parameter $t$ to the known RI dispersion of water ${ }^{19}$ at $20^{\circ} \mathrm{C}$ to an accuracy of within $10^{-6}$ RIU, as shown in Fig. 3(a). Here, the values used for $n_{2,3}\left(\lambda_{R}\right)$ were obtained from the dispersion data in Fig. 1(b) via interpolation. This resulted in a calibration curve of thickness $t$ vs. period $\Lambda$, shown in Fig. 3(b), with the obtained thicknesses all being in the range $28.4-28.7 \mathrm{~nm}$.

(a)

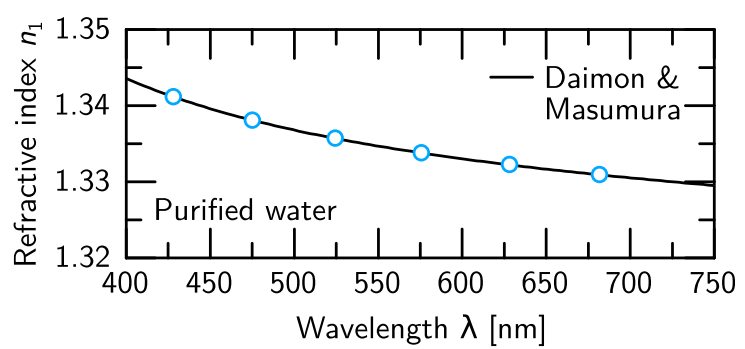

(b)

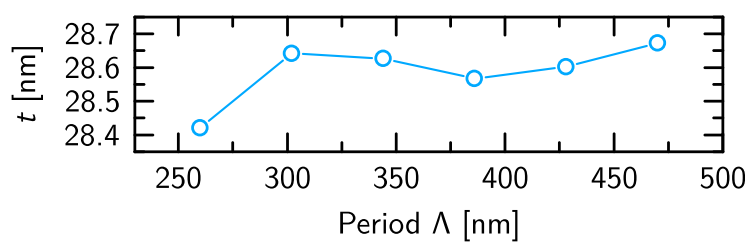

FIG. 3. (a) Datapoints obtained from measurements and Eq. (6), fitted to the dispersion curve of purified water at $20^{\circ} \mathrm{C}$ as reported by Daimon and Masumura. ${ }^{19}$ (b) The resulting calibration curve of thickness $t$ vs. period $\Lambda$.
The calibration curve, the dispersion data in Fig. 1(b) and Eq. (6) were then used in conjunction with the experimentally obtained resonance wavelengths $\lambda_{R}$ for the three remaining liquids in order to calculate their respective dispersion curves, shown in Fig. 4. The results for each material were then fitted to a Cauchy curve of the form $A+B \lambda^{-2}+C \lambda^{-4}$. In the case of the index matching oil and immersion oil, the obtained dispersion curves are compared to data provided by the manufacturer, whereas in the case of the glycerol, the results are compared to literature-published data. The results show clear agreement between the RI dispersion curves obtained by this method and the reference data, both in terms of the dispersion profile, and in terms of absolute RI values.

In the case of the two oils, the manufacturer's datapoints fall on the obtained Cauchy curve within error, apart from two: the first immersion oil datapoint and the second RI matching oil datapoint, which are off by $4 \times 10^{-4}$ RIU and $2 \times 10^{-4}$ RIU, respectively. In the case of glycerol, the dispersion as reported by Cooper runs in parallel to the obtained dispersion curve, but at higher values. However, as shown by Hoyt, ${ }^{22}$ the RI of glycerol is quite sensitive to its purity in terms of water content, with Cooper's results corresponding to a purity of $\sim 99.5 \%$, and the ones obtained with the present method being consistent with a purity of $98 \%-99 \%$. The purity of the glycerol used here was initially $99 \%$ when purchased, but is likely to have become reduced before use, due to, e.g., handling, refrigeration, and atmospheric contact, which would account for the difference. Finally, the obtained data agree generally with that of Birkhoff et al., although they only gave their data with two significant digits.

(a)

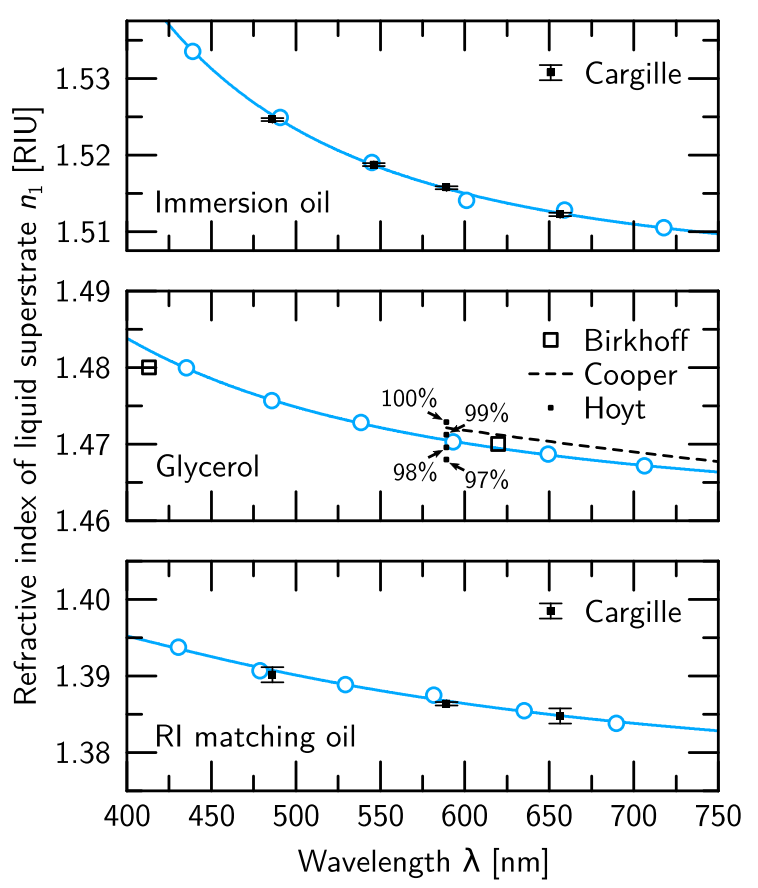

FIG. 4. The obtained dispersion curves using the presented method for the case of three different superstrate liquids: (a) Immersion oil, (b) glycerol, and (c) a RI matching oil. Open circles and solid lines denote datapoints obtained using this method and a corresponding Cauchy-fit, respectively. Other datapoints denote reference values from either material manufacturers (Cargille Labs) or literature (Birkhoff, ${ }^{20}$ Cooper, ${ }^{21}$ and $\mathrm{Hoyt}^{22}$ ). The data from Hoyt show the refractive indices of four different purities of glycerol in terms of water content. 
The state-of-the-art method for measuring the RI dispersion of liquids is, and has been since its inception two centuries ago, the Newton-Fraunhofer method of minimum deviation. Here, light is directed through a prism-shaped cell filled with a sample liquid at an angle of incidence such that the deflection caused by the prism is minimized, and by measuring the deflection angle, the refractive index of the liquid can be calculated. This method has an achievable precision of $1 \times 10^{-5}$ RIU, ${ }^{23}$ which is practically limited by the thermo-optic coefficient of the liquid and the degree to which the liquid can be thermally stabilized. For high-precision measurements, the RI dispersion of the air surrounding the prism and the prism cell material must be taken into account. The method presented in this work requires only a small amount of sample liquid $(100 \mu \mathrm{l})$ compared to that required for the method of minimum deviation $(\sim 70 \mathrm{ml})$, with a potential for further reduction by the implementing of a microfluidic system to bring the liquid to the PCRR array. As temperature plays a key role in the RI measurements of liquids, this may be beneficial for rapid dispersion measurements, since a much smaller volume must be thermally stabilized. The method can further be extended to measure at an arbitrary number of wavelengths, simply by increasing the number of distinct periods in the array. Since the optical resonance has no overlap with the ambient surroundings, there is no need for correcting the obtained results for the RI of air. Furthermore, this method is compact in nature, and is thus well suited for lab-on-a-chip applications or other usecases where space is scarce.

In conclusion, a method for measuring the refractive index dispersion of liquids using an array of photonic crystal resonant reflectors is presented. The method is demonstrated for the case of three different liquids and found to be in agreement with reference values, both in terms of absolute refractive index values and in terms of the dispersion profile. The method requires that the dispersion of the materials from which the PCRR array is composed must be known, as well as an initial calibration step with respect to a liquid of known dispersion, but this step may be easily automated using a commonly available liquid such as purified water. In any case, measuring dispersion as opposed to refractive indices at single wavelengths yields an additional dimension of information about the process being monitored, and offers the capability for monitoring multiple processes simultaneously. Further applications for dispersion sensing may also lie in rapid liquid identification and distinguishing between liquids which, e.g., may have the same RI at a particular wavelength but different dispersion profiles.

The authors acknowledge funding from the European Commission under the Seventh Framework Programme under Grant Agreement No. 278204 (Cellomatic) and from the Strategic Research Center PolyNano (10-092322). C.V. and C.L.C.S. acknowledge support from the Danish Research Council for Technology and Production Sciences (Grant Nos. 12-126676 and 12-126601).

${ }^{1}$ X. Fan, I. M. White, S. I. Shopova, H. Zhu, J. D. Suter, and Y. Sun, Anal. Chim. Acta 620, 8 (2008).

${ }^{2}$ S. Krey, D. Off, and A. Ruprecht, Proc. SPIE 8992, 89920D (2014).

${ }^{3}$ S. S. Wang, R. Magnusson, J. S. Bagby, and M. G. Moharam, J. Opt. Soc. Am. A 7, 1470 (1990).

${ }^{4}$ S. Fan and J. D. Joannopoulos, Phys. Rev. B 65, 235112 (2002).

${ }^{5}$ R. Magnusson and S. S. Wang, Appl. Phys. Lett. 61, 1022 (1992).

${ }^{6}$ S. S. Wang and R. Magnusson, Appl. Opt. 32, 2606 (1993).

${ }^{7}$ C. F. R. Mateus, M. C. Y. Huang, Y. Deng, A. R. Neureuther, and C. J. Chang-Hasnain, IEEE Photonics Technol. Lett. 16, 518 (2004).

${ }^{8}$ D. W. Dobbs and B. T. Cunningham, Appl. Opt. 45, 7286 (2006).

${ }^{9}$ Y. Ding and R. Magnusson, Opt. Express 12, 5661 (2004).

${ }^{10}$ B. T. Cunningham, P. Li, B. Lin, and J. Pepper, Sens. Actuators, B 81, 316 (2002).

${ }^{11}$ Y. Nazirizadeh, U. Bog, S. Sekula, T. Mappes, U. Lemmer, and M. Gerken, Opt. Express 18, 19120 (2010).

${ }^{12}$ Y. Nazirizadeh, J. Reverey, U. Geyer, U. Lemmer, C. Selhuber-Unkel, and M. Gerken, Appl. Phys. Lett. 102, 011116 (2013).

${ }^{13}$ W. Chen, K. D. Long, M. Lu, V. Chaudhery, H. Yu, J. S. Choi, J. Polans, Y. Zhuo, B. A. C. Harley, and B. T. Cunningham, Analyst 138, 5886 (2013).

${ }^{14}$ C. Vannahme, C. L. C. Smith, M. B. Christiansen, and A. Kristensen, Appl. Phys. Lett. 101, 151123 (2012).

${ }^{15}$ P. G. Hermannsson, C. Vannahme, C. L. C. Smith, and A. Kristensen, Appl. Phys. Lett. 105, 071103 (2014).

${ }^{16}$ A. Yariv and P. Yeh, Photonics: Optical Electronics in Modern Communications, 6th ed. (Oxford University Press, 2006).

${ }^{17}$ G. E. Jellison and F. A. Modine, Appl. Phys. Lett. 69, 2137 (1996).

${ }^{18}$ G. A. Turnbull, P. Andrew, M. J. Jory, W. L. Barnes, and I. D. W. Samuel, Phys. Rev. B 64, 125122 (2001).

${ }^{19}$ M. Daimon and A. Masumura, Appl. Opt. 46, 3811 (2007).

${ }^{20}$ R. D. Birkhoff, L. R. Painter, and J. M. Heller, J. Chem. Phys. 69, 4185 (1978).

${ }^{21}$ P. R. Cooper, Appl. Opt. 22, 3070 (1983).

${ }^{22}$ L. F. Hoyt, Ind. Eng. Chem. 26, 329 (1934).

${ }^{23}$ The National Metrology Institute of Germany, private communication (2015). 